\title{
Center for Advanced Materials
}

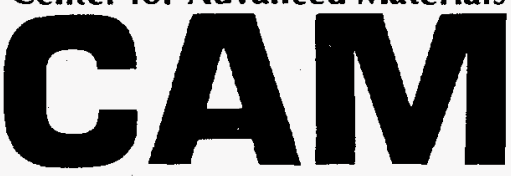

Presented at the Seventeenth International Conference on

Defects in Semiconductors, Gmunden, Austria, July 18-23, 1993,

and to be published in Materials Science Forum

Scanning Tunneling Microscopy of Si Donors in GaAs

RECEIVED

J.-F. Zheng, X. Liu, N. Newman, E.R. Weber, D.F. Ogletree, and M.B. Salmeron

July 1993

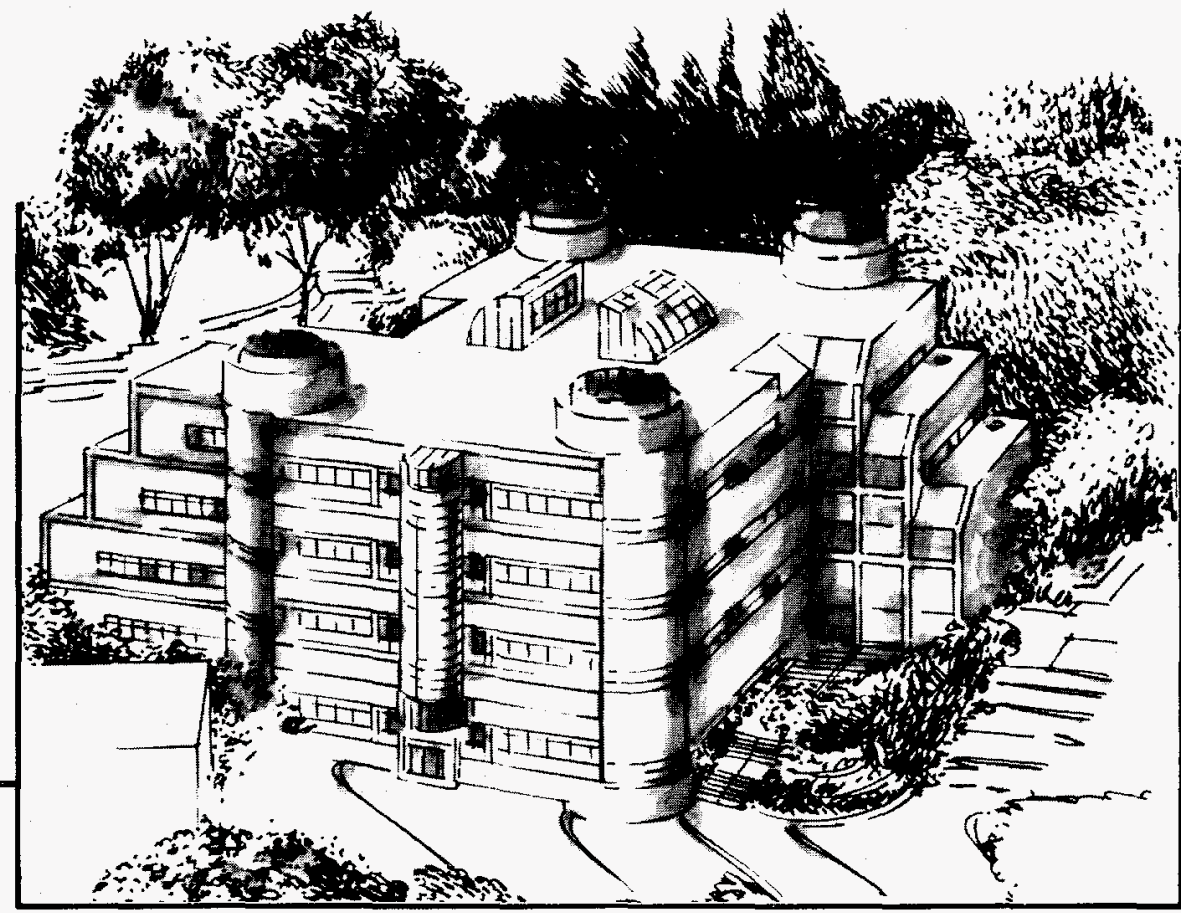

\section{Materials and Chemical Sciences Division}

\section{Lawrence Berkeley Laboratory - University of California}

ONE CYCLOTRON ROAD, BERKELEY, CA 94720 •(415) 486-4755 


\section{DISCLAIMER}

This document was prepared as an account of work sponsored by the United States Government. Neither the United States Government nor any agency thereof, nor The Regents of the University of California, nor any of their employees, makes any warranty, express or implied, or assumes any legal liability or responsibility for the accuracy, completeness, or usefulness of any information, apparatus, product, or process disclosed, or represents that its use would not infringe privately owned rights. Reference herein to any specific commercial product, process, or service by its trade name, trademark, manufacturer, or otherwise, does not necessarily constitute or imply its endorsement, recommendation, or favoring by the United States Government or any agency thereof, or The Regents of the University of California. The views and opinions of authors expressed herein do not necessarily state or reflect those of the United States Government or any agency thereof or The Regents of the University of California and shall not be used for advertising or product endorsement purposes.

Lawrence Berkeley Laboratory is an equal opportunity employer. 


\section{DISCLAIMER}

Portions of this document may be illegible in electronic image products. Images are produced from the best available original document. 
LBL-34499

UC-411

\title{
Scanning Tunneling Microscopy of Si Donors in GaAs
}

\author{
J.-F. Zheng, ${ }^{1,2}$ X. Liu, ${ }^{1}$ N. Newman, ${ }^{1}$ E.R. Weber, ${ }^{1,2}$ \\ D.F. Ogletree, ${ }^{2}$ and M.B. Salmeron ${ }^{2}$ \\ 1Department of Materials Science \\ University of California, Berkeley \\ Berkeley, California 94720 \\ 2Materials Science Division \\ Lawrence Berkeley Laboratory \\ University of California \\ Berkeley, California 94720
}

July 1993

This work was supported by the Director, Office of Energy Research, Office of Basic Energy Research, Materials Sciences Division, of the U.S. Department of Energy under Contract No. DE-AC03-76SF00098. 


\title{
Scanning tunneling microscopy of Si donors in GaAs
}

\author{
J.-F. Zheng 1,2, X. Liu, N. Newman, and E.R. Weber 1,2 \\ 1Department of Materials Science, University of California at Berkeley, \\ Berkeley, CA 94720 \\ D.F. Ogletree and M.B. Salmeron \\ 2Materials Science Division, Lawrence Berkeley Laboratory, Berkeley, CA 94720
}

Keywords: STM, surface state, hydrogenic impurity, GaAs

\begin{abstract}
Using scanning tunneling microscopy, we have identified and characterized $\mathrm{Si}$ donors $\left(\mathrm{Si}_{\mathrm{Ga}}\right)$ in $\mathrm{GaAs}$ located on the (110) surface and in subsurface layers. $\mathrm{SiGa}_{\mathrm{Ga}}$ on the surface shows localized features with characteristic structures in good agreement with a recent theoretical calculation [Wang et al., Phys. Rev. B, 47, 10329 (1993)]. Si Ga in subsurface layers appears as delocalized protrusions superimposed on the background lattice, which are interpreted in terms of the modification of the tunneling due to the tip-induced band bending perturbed by the $\mathrm{Si}_{\mathrm{Ga}}$ Coulomb potential.
\end{abstract}

\section{Introduction}

The power of scanning tunneling microscopy (STM) for the study of geometric and electronic structure of semiconductor surfaces has long been realized [1, 2]. Recently, the use of STM to investigate the bulk defect properties of semiconductors has been beautifully demonstrated by the study of antisite defects in low-temperature-grown GaAs [3]. The properties of an imperfection in 
a bulk semiconductor are often distinctly different from those on a surface, and STM studies of these imperfections will definitely become more important in the future, although up to the present, few such studies have been reported.

Shallow substitutional impurities, such as $\mathrm{Si}$ donors $\left(\mathrm{Si}_{\mathrm{Ga}}\right)$ in $\mathrm{GaAs}$, are probably among the best known and best understood imperfections in semiconductors. In the bulk, the electronic structures of these impurities are known to be delocalized and well described by the hydrogenic model. At the surface, broken bonds of these impurities may introduce strongly localized electronic structures and deep states near the middle of the energy gap. In a recent theoretical study, Wang et al. [4] predicted that STM images of SiGa on a GaAs (110) surface should reveal unique, localized electronic structures in voltage-dependent images and current-voltage (I-V) spectra. Experimentally, these localized $\mathrm{Si}_{\mathrm{Ga}}$ features have never been reported, although Feenstra et al. observed fluctuations due to Si on GaAs (110) surfaces in a STM study of doping superlattices [5].

In this work, we performed STM studies on (110) studies of Si-doped GaAs crystals. Localized electronic features were clearly observed. The filledand empty-state STM images show characteristic electronic structures in good agreement with the theoretical predictions of the $\mathrm{Si}_{\mathrm{Ga}}$ on the GaAs (110) surface. In addition to these localized structures, Si-related protrusions superimposed on the background atomic lattice were also observed, and we interpret these protrusions in terms of the modification of the tunneling due to the tip-induced band bending perturbed by the $\mathrm{Si}_{\mathrm{Ga}}$ Coulomb potential.

\section{Experiments}

Si doped GaAs crystals grown by liquid encapsulated Czochraski technique were studied. Samples from different sources, and with high and low 
Si doping concentrations, $\mathrm{n}^{+}$-GaAs $\left(2 \times 10^{18} / \mathrm{cm}^{3}\right)$ and $\mathrm{n}-\mathrm{GaAs}\left(5 \times 10^{16} / \mathrm{cm}^{3}\right)$, were investigated. The STM head was similar to that developed by J. Frohn et al. [6], which allows the tip to move over a large area of the sample surface. The electronic control was a commercial unit made by RHK [7]. The samples were cleaved in-situ in a ultra-high-vacuum chamber with a base pressure of $8 \times 10^{-11}$ torr. Electrochemically etched Pt-Rh tips were used. All STM images were obtained in constant current mode with a tunneling current of $0.5 \mathrm{nA}$.

\section{Results and discussion}

Localized features observed on the Si-doped GaAs (110) surface (Fig. 1) are attributed to $\mathrm{Si}_{\mathrm{Ga}}$ donor atoms situated on the cleavage plane based on the theoretical work of Wang et al. [4]. The most characteristic images are obtained for sample voltages of $-2.5 \mathrm{~V}$ (Fig. 1a) and $+2.5 \mathrm{~V}$ (Fig. 1b), corresponding to filled- and empty-state images, and matching very well with the theoretically predicted images of a $\mathrm{Si}_{\mathrm{Ga}}$ on $\mathrm{GaAs}(110)$ surface at voltages of $-1.2 \mathrm{~V}$ and $+1.9 \mathrm{~V}$, respectively. The theoretical calculation did not consider tip-induced band bending, and assumed a Fermi level at midgap. By taking the band bending into account, and considering that in our sample the Fermi level is at the conduction band edge, the experimental images at -2.5 and $+2.5 \mathrm{~V}$ should closely correspond to the theoretical images at -1.2 and $+1.9 \mathrm{~V}$, respectively. We believe this is the first direct observation of surface $\mathrm{Si}_{\mathrm{Ga}}$.

The localized features in Fig. 1 are readily understood if modifications to the electronic structures of the GaAs (110) surface are considered. According to Wang et al. [4], when a $\mathrm{Si}_{\mathrm{Ga}}$ donor atom is on the surface, a state near midgap is introduced due to the dangling bonds of the atom. Since the dangling-bond state has a lower energy than the hydrogenic state of the $\mathrm{SiGa}_{\mathrm{Ga}}$ donor in bulk $\mathrm{GaAs}$, the extra electron of the $\mathrm{Si}_{\mathrm{Ga}}$ is thus trapped in this localized, lower energy state. In 
addition, this midgap state interacts with the surface dangling-bond states of neighboring As and $\mathrm{Ga}$. The resulting consequences are: (a) the dangling-bond state of the $\mathrm{Si}_{\mathrm{Ga}}$ will have an energy slightly above midgap; (b) the danglingbond states of As atoms adjacent to the $\mathrm{Si}_{\mathrm{Ga}}$ are pushed to lower energies than their normal positions near the valence bond edge; and (c) the dangling-bond states of $\mathrm{Ga}$ atoms adjacent to the $\mathrm{Si}_{\mathrm{Ga}}$ are also slightly pushed to higher energies than their normal positions near the conduction band edge. The above effects are expected to be localized in the neighborhood of the $\mathrm{Si}_{\mathrm{Ga}}$ since the dangling-bond state of the $\mathrm{SiGa}_{\mathrm{Ga}}$ is localized to within a few bond lengths.

For a sample voltage of $-2.5 \mathrm{~V}$, as seen in Fig. 1a, the tunneling condition has been optimized for electrons to tunnel out of the filled, normal danglingbond states of As atoms not in the neighborhood of the $\mathrm{Si}_{\mathrm{Ga}}$. At this tunneling condition, electrons can also tunnel out of the half-filled, dangling-bond state of the $\mathrm{Si}_{\mathrm{Ga}}$. A local maximum is thus observed at the position of the $\mathrm{SiGa}_{\mathrm{Ga}}$. Since the filled, dangling-bond states of the As atoms immediately adjacent to the SiGa lie at lower energies, the voltage of $-2.5 \mathrm{~V}$ is not sufficient for electrons to tunnel out of these states. A denuded zone of apparently missing As atoms are therefore observed at the positions of the two As atoms bonded to the SiGa. Note in Fig. 1a that the two As atoms neighboring the apparently missing As atoms, however, appear brighter, which was not predicted in the theoretical work. At a sample voltage of $+2.5 \mathrm{~V}$ (Fig. 1b), the image of the surface $\mathrm{SiGa}_{\mathrm{G}}$ appears significantly different. Electrons tunnel at this voltage from the tip into the dangling-bond states of the $\mathrm{Ga}$ atoms. The Ga sublattice on GaAs (110) surface is clearly observed. However, the tunneling into the dangling-bond state of the $\mathrm{Si}$ atom is weaker, since the state is half filled. The SiGa thus appears dark in the image and resembles a missing $\mathrm{Ga}$ atom. The $\mathrm{Ga}$ atoms neighboring the $\mathrm{Si}$ atom also appear dark in the image, since the dangling-bond states of these $\mathrm{Ga}$ atoms lie at higher 
energies due to the interaction with the $\mathrm{Si}_{\mathrm{Ga}}$ dangling-bond state, and it is more difficult for electrons to tunnel into these states.

An interesting observation for the localized surface $\mathrm{Si}_{\mathrm{Ga}}$ feature is that their experimentally measured density is lower than what one would expect from the $\mathrm{Si}$ doping concentration of the sample. For instance, for $\mathrm{Si}$ doping concentration of $2 \times 10^{18} / \mathrm{cm}^{3}$, the expected density of the $\mathrm{Si}_{\mathrm{Ga}}$ on a (110) plane would be $4 \times 10^{10} / \mathrm{cm}^{2}$. The reason for the much fewer SiGa features observed is not fully clear yet. It is, however, suspected to be due to the instability of the SiGa having half-filled dangling bonds on a cleavage plane. The SiGa may have left the crystal during cleavage.

In addition to the localized electronic features associated with the $\mathrm{Si}_{\mathrm{Ga}}$ on the (110) surface, delocalized features (Fig. 2) are also observed. They appear as protrusions superimposed on the background atomic lattice for both the positive and negative sample voltages. These features are believed to be due to subsurface $\mathrm{Si}$ since their concentration scales well with the $\mathrm{Si}$ doping concentration. Images in Fig. 2 show that the apparent height of the protrusions is strongly bias dependent, and in most cases decreases from $\sim 1 \AA$ at $-1.5 \mathrm{~V}$ to $\sim 0.1 \AA$ at $-3.0 \mathrm{~V}$. The spatial extension of the protrusions decreases slowly with increasing negative sample voltages. The decrease of the extension of the protrusions at increasing positive voltages, however, is less significant. Typically, the spatial extension of the protrusions is $\sim 50 \AA$ ( $25 \AA$ at full width half maximum) at $-1.5 \mathrm{~V}$, and decreases to about half that value at $-3.0 \mathrm{~V}$. We believe these delocalized protrusions are due to $\mathrm{Si}_{\mathrm{Ga}}$ in bulk $\mathrm{GaAs}$ located in subsurface layers below the (110) cleavage plane. In fact, the apparent height of the protrusions measured at the same sample voltage is statistically found to have several discrete values, and are attributed to $\mathrm{SiGa}_{\mathrm{G}}$ atoms situated in subsurface layers with different distances to the cleavage plane [8]. 
The delocalized protrusions of a $\mathrm{Si}_{\mathrm{Ga}}$ in a subsurface layer observed for both positive and negative sample voltages are interpreted in Fig. 3 in terms of the modification of the tunneling due to the tip-induced band bending perturbed by the $\mathrm{Si}_{\mathrm{Ga}}$ Coulomb potential $[9,10]$. At positive sample voltages (Fig. $3 \mathrm{a}$ ), electrons tunnel from the metal tip to the empty conduction band states in the semiconductor. In such conditions, the GaAs subsurface region is expected to be in depletion in analogy to metal-insulator-semiconductor contacts. The $\mathrm{Si}_{\mathrm{Ga}}$ in the depletion region is positively charged. The Coulomb potentials of the $\mathrm{SiGa}$ $(\sim 1 / \mathrm{r})$, with their overlapping tails, give rise to the average band bending in the semiconductor. Close to the core of each of the $\mathrm{Si}_{\mathrm{Ga}}$ the Coulomb potential is stronger, which locally decreases the band bending and significantly increases the available states for tunneling. The observed radius of the protrusions $(\sim 25 \AA)$ is consistent with the extension of the dominant part of the Coulomb potential (defined as the region where the potential is stronger than $\mathrm{kT} \sim 26 \mathrm{meV}$ ). At negative sample voltages (Fig. $3 b$ ), electrons tunnel from the valence-band states and filled conduction-band states of the semiconductor to the metal tip. In such conditions, the GaAs subsurface region can be brought into accumulation, and the $\mathrm{Si}_{\mathrm{Ga}}$ is expected to be neutral. However, the potential near the core of the

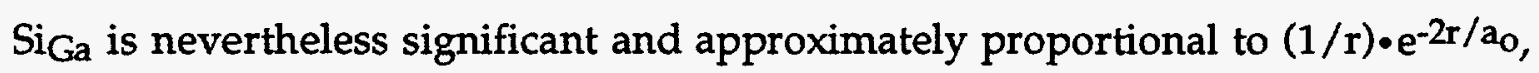
where $\mathrm{a}_{\mathrm{o}} \sim 85 \AA$ is the Bohr radius of the hydrogenic donors in GaAs. Close to the core of the $\mathrm{SiGa}_{\mathrm{G}}$, the potential still locally decreases the band energy, resulting in stronger tunneling and hence the observed protrusions. Obviously, the dominant part of the potential (at $\mathrm{r} \leq 25 \AA<\mathrm{a}_{0} / 2$ ) is only slightly different from that of the $1 / \mathrm{r}$ potential in depletion. Hence the radius of the protrusions is expected to be $\sim 25 \AA$, which is consistent with the experimental observations at sample voltage of $-1.5 \mathrm{~V}$.

The decrease of the apparent height of the protrusions is expected at both 
higher positive and negative sample voltages, since the tunneling is then dominated by a large number of band states available, and is less sensitive to the presence of the $\mathrm{SiGa}_{\mathrm{Ga}}$. The slow decrease of the extension of the protrusions with increasing negative sample voltages may be attributed to the screening of the $\mathrm{Si}_{\mathrm{G} a}$ potential by conduction electrons accumulated close to the surface. In bulk GaAs, the calculated characteristic screening length is $\sim 55 \AA$ for an electron concentration of $10^{18} / \mathrm{cm}^{3}$, and $\sim 25 \AA$ for an electron concentration of $10^{20} / \mathrm{cm}^{3}$ [11]. The screening by a thin layer of electrons, however, is known to be less effective, and the screened Coulomb potential is proportional to $\sim 1 / \mathrm{r}^{3}$ in a 2dimensional limit in contrast to $\sim(1 / \mathrm{r}) \bullet \mathrm{e}^{-\mathrm{r} / \mathrm{L}}$ in a 3-dimensional case [12]. The characteristic screening length in the limit of degenerate 2-dimensional electron gas in GaAs is calculated to be $\sim 45 \AA$, independent of the electron density $[13,14]$. The experimentally observed decrease of the extension of the protrusions, from $\sim 50 \AA$ at $-1.5 \mathrm{~V}$ in weak accumulation to a minimum of about half that value at $-3.0 \mathrm{~V}$ in strong accumulation, is thus in good agreement with what one would expect from the effect of screening by conduction electrons. For positive sample voltages, no such screening is expected since the surface is depleted of electrons, which is consistent with the experimental observation that the decrease of the extension of the protrusions is less significant with increasing positive sample voltages.

In conclusion, for the first time we have studied by scanning tunneling microscopy both the localized electronic features associated with the danglingbond states of $\mathrm{Si}$ donors $\left(\mathrm{Si}_{\mathrm{Ga}}\right)$ situated on $\mathrm{GaAs}(110)$ surface, and the delocalized electronic features associated with the Coulomb potential of the $\mathrm{Si}_{\mathrm{Ga}}$ in bulk GaAs located below the (110) cleavage surface. 
Acknowledgments: The authors are grateful to Dr. W. Walukiewicz for helpful discussions. This work was supported by the Director, Office of Energy Research, Office of Basic Energy Research, Materials Science Division, U.S. Department of Energy under contract no. DE-AC03-76SF00098. 


\section{REFERENCES}

1. G. Binnig, H. Rohrer, C. Gerber, E. Weibel, Phys. Rev. Lett., 50, 120 (1983).

2. J. A. Stroscio and J. William, Scanning Tunneling Microscopy, in Methods in Experimental Physics, V. 27 (Academic Press, Boston, 1993), and references therein.

3. R. M. Feenstra, J. M. Woodall, and G.D. Pettit, Phys. Rev. Lett., 71, 1176 (1993).

4. J. Wang, T. A. Arias, and J. D. Joannopoulos, Phys. Rev. B, 47, 10329 (1993).

5. R. M. Feenstra, E. T. Yu, J. M. Woodall, P. D. Kirchner, C. L. Lin, and G. D. Pettit, Appl. Phys. Lett., 61, 795 (1992).

6. J. Frohn, J. F. Wolf, K. H. Besocke, and N. Teske, Rev. Sci. Instrum., 60, 1200 (1989).

7. RHK Technology, Inc., Rochester Hills, Michigan.

8. J.-F. Zheng, X. Liu, E. R. Weber, D. F. Ogletree, and M. B. Salmeron, unpublished.

9. R. M. Feenstra, and J. A. Stroscio, J. Vac. Sci. Technol. B, 5, 923 (1987).

10. R. Maboudian, K. Pond, V. Bressler-Hill, M. Wassermeier, P. M. Petroff, G. A. D. Griggs, and W. H. Weinberg, Surf. Sci. Letts., 275, L662 (1992).

11. R. B. Dingle, Philos. Mag., 46, 861 (1955).

12. T. Ando, A. B. Fowler, and F. Stern, Rev. Mod. Phys., 54, 437 (1982).

13. F. Stern, Phys. Rev. Lett., 18, 546 (1967).

14. F. Stern, and W. E. Howard, Phys. Rev., 163, 816 (1967). 


\section{FIGURE CAPTIONS}

Fig. 1 STM topographic images of a Si $\mathrm{Ga}$ on $\mathrm{GaAs}$ (110) cleavage surface. (a) Filled state STM image acquired at $-2.5 \mathrm{~V}$ sample bias. (b) Empty state STM image acquired at $+2.5 \mathrm{~V}$ sample bias. The locations of the $\mathrm{SiGa}_{\mathrm{Ga}}$ is marked by the arrows in the images.

Fig. 2 STM images of the delocalized protrusions arising from subsurface $\mathrm{Si}_{\mathrm{Ga}}$ taken with a tunneling current of $0.5 \mathrm{nA}$ and at sample voltages of (a) $-3.0 \mathrm{~V}$; (b) $-1.5 \mathrm{~V}$. The topographic profile across the features along the line of the arrows in (a) and (b) is shown in (c). With increasing negative sample voltages, the apparent height and the spatial extension of the protrusions decreases.

Fig. 3 Schematic illustrations of the energy band diagrams and the electron tunneling processes. (a) at a positive sample voltage; (b) at a negative sample voltage. The dash lines in the figure illustrate the band perturbed by the subsurface $\mathrm{Si}_{\mathrm{Ga}}$. 


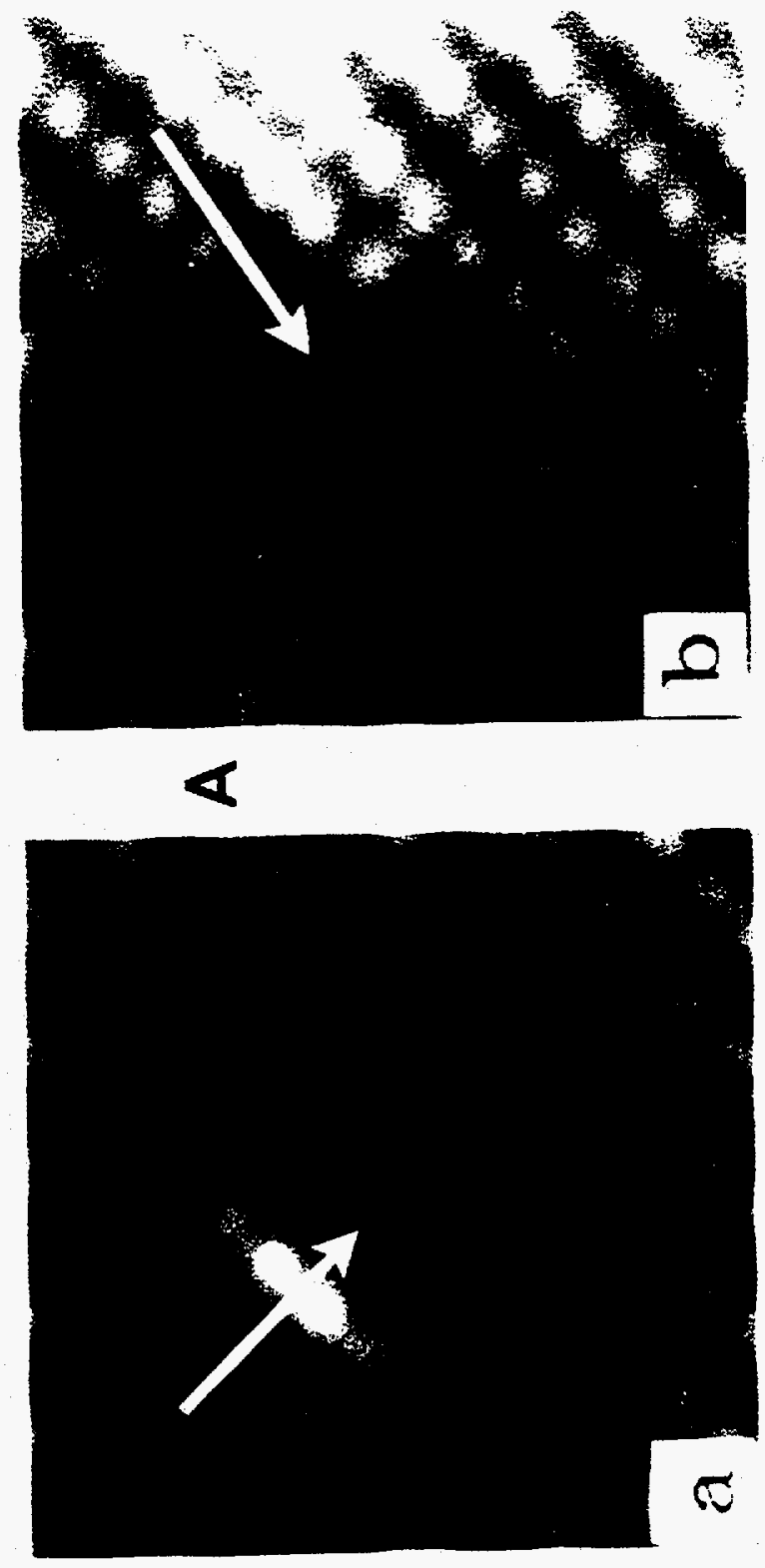

Di 

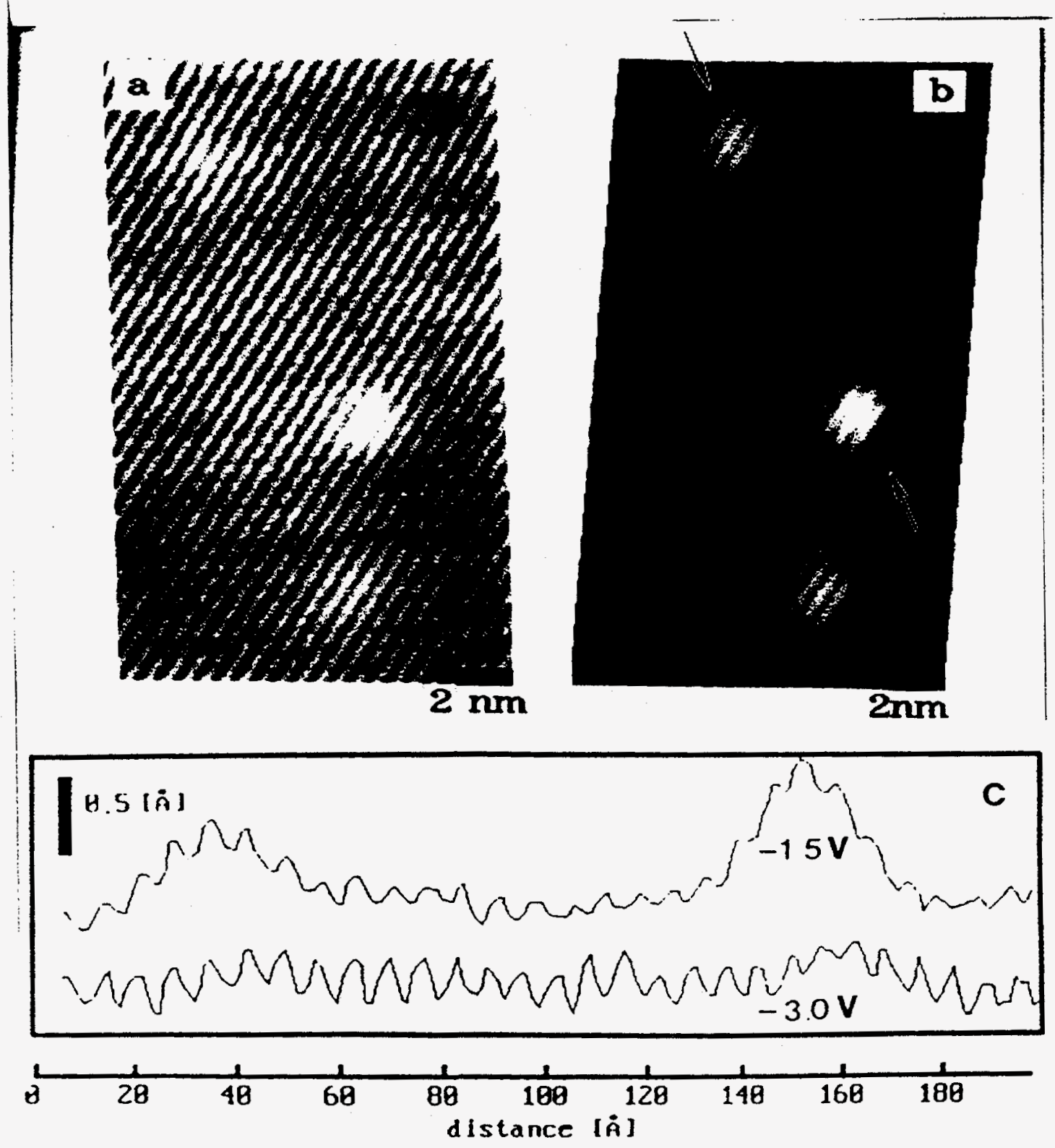

Fig. 2 


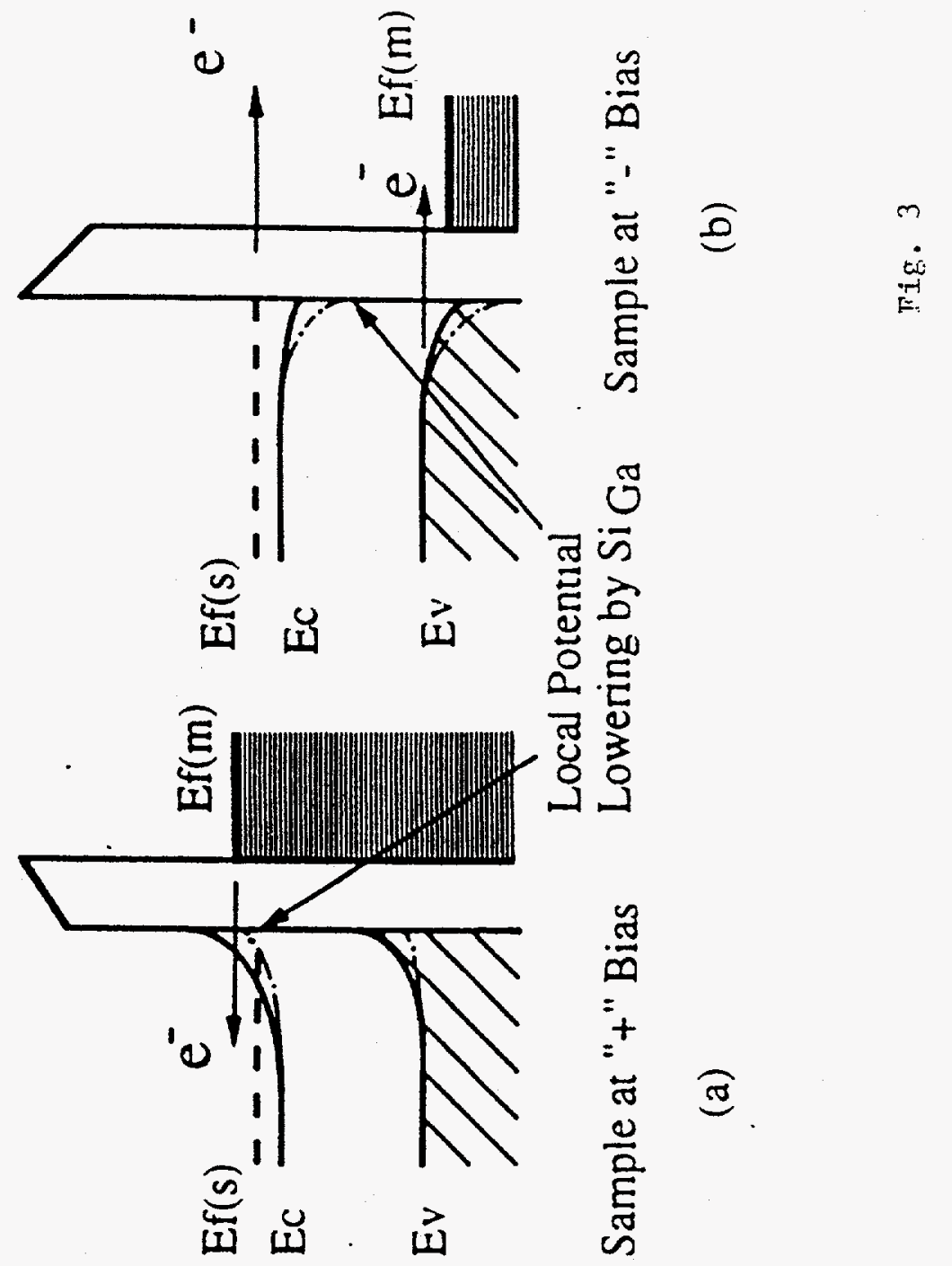

